\title{
Perioperative Interstitial Brachytherapy as a Boost in Breast Cancer Conserving Therapy
}

\author{
Dominique J. P. van Uden ${ }^{1 *}$, Elzbieta M. van der Steen-Banasik ${ }^{2}$, Manuel J. Koppe ${ }^{1}$, Marcel R. Stam ${ }^{2}$, \\ Jan van Wijk ${ }^{3}$, Charlotte F. J. M. Blanken-Peeters ${ }^{1}$ \\ ${ }^{1}$ Department of Surgery, Rijnstate Hospital, Arnhem, The Netherlands; ${ }^{2}$ Arnhem Radiotherapy Institute, Arnhem, The Netherlands; \\ ${ }^{3}$ Department of Surgery, Zevenaar Hospital, Zevenaar, The Netherlands. \\ Email: *djpvanuden@gmail.com
}

Received July $8^{\text {th }}, 2012$; revised August $10^{\text {th }}, 2012$; accepted August $21^{\text {st }}, 2012$

\begin{abstract}
Background: The aim of breast-conserving treatment (BCT) in breast cancer, consisting of wide local excision (WLE) and radiotherapy, is to obtain local control of disease as well as an optimal cosmetic result. The application of perioperative brachytherapy allows more precise deposition of the boost radiation dose to the lumpectomy cavity plus margins in a shorter period of time, as compared to external beam radiotherapy. The aim of the present study was to analyze the oncological outcome of interstitial brachytherapy in our patient population of patients with early-stage breast cancer. Patients and Methods: 107 breasts in 105 patients with T1-2 breast cancer were treated between 1996 and 2009 with BCT, including WLE combined with perioperative brachytherapy using Iridium-192 (15 Gy with low dose rate or 9 Gy with high dose rate) followed by whole breast irradiation (50 Gy). Outcomes analyzed included treatment toxicity (according to Common Terminology Criteria for Adverse Events), local recurrence rate, and disease-free and overall survival. Results: Median follow-up of patients still alive was 6.3 years (range 2.0 - 14.5). Mean age was 50.2 years $(+/-$ S.D. 10.5). Mean tumor diameter was $15 \mathrm{~mm}(+/-$ S.D. $8 \mathrm{~mm}$. Acute complications consisted of grade 1 and 3 complications, respectively $n=8$ and $n=1$. Late complications consisted of grade 1 or 2 , respectively $\mathrm{n}=25$ and $\mathrm{n}=2$. Only three patients $(2.8 \%)$ developed a local recurrence with a median time to recurrence of 9.3 years (range 3.3 - 9.3). Five- and ten-year local recurrence free survival was $99 \%$ and $91 \%$, respectively. Five- and ten-year disease-specific and overall survival was $95 \%$ and $84 \%$ versus $92 \%$ and $78 \%$, respectively. Conclusions: Treatmentrelated toxicity after brachytherapy for breast cancer was mild. The local recurrence rate is low. Therefore, brachytherapy is a good alternative to conventional radiation boost as a part of breast irradiation in breast conserving treatment in early stage breast cancer.
\end{abstract}

Keywords: Breast Cancer; Breast Conserving Treatment; Perioperative Interstitial Brachytherapy

\section{Introduction}

Wide local excision (WLE) and radiotherapy aim to achieve a good cosmetic result and to obtain locoregional control of disease in breast-conserving treatment (BCT) in breast cancer. Radiotherapy consists of the whole breast irradiation with an additional boost to the tumor site. Since the early nineties of the last century, BCT has been widely accepted as a treatment option for breast cancer. Still, whereas survival following BCT is similar to that obtained after ablative surgery, local recurrence is unfortunately still an issue. Several risk factors for local recurrence have been identified, including younger age, multifocality, the presence of ductal carcinoma in situ (DCIS), tumor-positive resection margins, and higher histological grade [1-5].

*Corresponding author.
Because the majority of local recurrences after breastconserving surgery occur close to the original tumor bed, the whole breast radiation is supplemented with a boost on the original tumor bed [6,7]. The boost radiation dose can be applied either with external beam, fractionated or with brachytherapy. The application of the boost by means of brachytherapy has an advantage of a precise application of the radiation dose to the lumpectomy cavity. With the introduction of brachytherapy, the radiation dose can be delivered over a shorter period of time as compared to conventional radiation schemes [8]. Another advantage of brachytherapy is a decreased radiation dose to the intrathoracic organs [9].

The role of the boost is constantly a subject of a discussion and consecutive clinical studies, however we do not consider this discussion a part of our study and thus will not comment on it anymore in this paper. 
The purpose of the present study was to analyze our results of perioperatively placed needles for remote afterloading Iridium-192 based brachytherapy in patients with early-stage breast cancer.

\section{Patients and Methods}

Between 1996 and 2009, we evaluated 107 breasts in 105 patients with early-stage breast cancer who were treated with breast-conserving therapy, including wide-local excision (lumpectomy) and a combination of Iridium-192 based brachytherapy and whole breast irradiation. Patients were selected based on the database according to the American Joint Committee on Cancer and the International Union for Cancer Control (AJCC-UICC) TNM breast cancer staging system: cT1 and cT2.

Of the 105 patients, two patients had bilateral breast cancers, which were primarily treated according to the same treatment protocol. In 40 patients (38\%) the decision to include brachytherapy in the radiation treatment was taken after the wide local excision and axillary staging operation. These patients underwent a second operation, during which the lumpectomy wound was opened and the needles were inserted. The brachytherapy and whole-breast irradiation were given at the Arnhem Radiotherapy Institute (ARTI). From the hospital files the following clinical and pathological characteristics were extracted: age, tumor size, pTNM-stage, tumor grade, estrogen-progesterone receptor status, the type of adjuvant systemic therapy, and time till locoergional or distant recurrence or death. Clinical and pathological TNM stages were determined according to the latest TNM classification of the Union for International Cancer Control (UICC) [10]. Complications were scored according to the Common Terminology Criteria for Adverse Events (CTCAE) [11]. Survival data was obtained for all patients. Data on tumor status at the time of death was complete.

\subsection{Surgery}

Surgical treatment consisted of wide-local excision. Until 2000 axillary staging consisted of formal axillary lymph node dissection, whereas from 2000 onwards patients underwent sentinel lymph node biopsy and formal axillary lymph node dissection, only in case of node-positive disease.

\subsection{Brachytherapy}

The needles were intraoperatively placed allowing precise coverage of the clinical tumor volume (CTV) plus a $1-2 \mathrm{~cm}$ margin of surrounding breast tissue. The hollow needles were connected to $\mathrm{m}$-selectron and Iridium 192 source was remotely inserted. Seven, nine or eleven metal needles were positioned in two layers in the lumpectomy cavity by a radiation oncologist using a plastic template to ensure even spacing. Subsequently, the lumpectomy wound was carefully closed in layers, thus minimizing the volume of the tumor bed.

As soon as postoperatively allowed, the patients were transported to the radiation oncology department. In the meantime, the target dose was calculated according to the rules of the Paris system [12]. The treatment started immediately and after completing of the irradiation the needles were removed. No extra anesthesia was necessary. From 1996 till 1999 brachytherapy consisted of a total of $15 \mathrm{~Gy}$, delivered at a low dose rate of $60 \mathrm{cGy}$ per hour (n = 17). From 1999 until the end of the study brachytherapy consisted of 9 Gy was delivered at a high dose rate When the wound had healed completely, all patients were treated with whole breast irradiation, according to the local protocol (50 Gy, 2 Gy per fraction for 5 weeks).

\subsection{Statistical Analysis}

Follow-up was measured from the date of surgery till the date of recurrence, death or last follow-up. The descriptive evaluation of data was performed using the statistical package SPSS for Windows, version 16.0 (SPSS Inc., Chicago, IL). Actuarial survival rates were calculated using the Kaplan-Meier method.

\section{Results}

Patient-related, surgical and pathological characteristics are given in Table 1. It shows that tumors were relatively small (mean diameter: $15 \mathrm{~mm}$, S.D. $8 \mathrm{~mm}$ ). In 22 patients $(21 \%)$ tumors were nonpalpable requiring wireguided resection. Of the 65 patients who underwent WLE and interstitial brachytherapy in one session, 5 patients (7.7\%) had tumor-positive resection margins. These patients were subsequently treated with ablative surgery. 44 Patients $(41 \%)$ had nodal disease, necessitating axillary lymph node dissection.

Median treatment volume of brachytherapy was 47.1 $\mathrm{cm}^{3}$ (range 23 - 114) with a median interval between brachytherapy and WBI of 28 (range $14-100$ ) days.

\subsection{Complications}

Acute complications consisted of wound infections of the lumpectomy site ( $\mathrm{n}=3 ; 2.8 \%$, CTCAE grade 1$)$, haematoma $(n=3 ; 2.8 \%$, grade 1$)$, venous bleeding due to removal of catheters, necessitating surgical exploration (n $=1 ; 0.9 \%$, grade 3$)$, wound infection of the axillary wound $(n=6 ; 5.6 \%$, grade 2$)$, pain $(n=1 ; 0.9 \%$, grade 1$)$ and postirradiation sterile mastitis $(\mathrm{n}=2 ; 1.9 \%$, grade 1$)$. Excluding the axillary wound infections, the overall 
Table 1. Clinicopathological characteristics of the 107 breast cancers in 105 patients. $T=$ tumor; $N=$ node; $E R=$ estrogen receptor; $\mathbf{P R}=$ progesterone receptor.

\begin{tabular}{|c|c|}
\hline \multicolumn{2}{|c|}{ Clinicopathological characteristics } \\
\hline Age (years, range) & $50.2(30-80)$ \\
\hline Follow up (mean in yr, range) & $6.7(0.5-14.8)$ \\
\hline \multicolumn{2}{|l|}{ Adjuvant therapy } \\
\hline Hormone therapy & $12(11.2)$ \\
\hline Chemotherapy & $54(49.5)$ \\
\hline None & $38(35.5)$ \\
\hline Unknown & $3(2.8)$ \\
\hline Tumor size (mm) & 15 (range $1-48$ ) \\
\hline $1-20$ & $85(80 \%)$ \\
\hline $21-30$ & $18(17 \%)$ \\
\hline $30<$ & $4(3 \%)$ \\
\hline \multicolumn{2}{|l|}{ Margins } \\
\hline$<2 \mathrm{~mm}$ & $7(7 \%)$ \\
\hline$>2 \mathrm{~mm}$ & $56(52 \%)$ \\
\hline$<1 \mathrm{~mm}$ & $11(10 \%)$ \\
\hline Irradical & $18(17 \%)$ \\
\hline Unknown & $15(14 \%)$ \\
\hline \multicolumn{2}{|l|}{ Pathologic T stage } \\
\hline $\mathrm{T}_{1}$ & $66(62 \%)$ \\
\hline $\mathrm{T}_{2}$ & $41(38 \%)$ \\
\hline \multicolumn{2}{|l|}{ Pathologic N stage } \\
\hline $\mathrm{N}_{\mathrm{x}}$ & $61(57 \%)$ \\
\hline $\mathrm{N}_{1}$ & $40(37 \%)$ \\
\hline $\mathrm{N}_{2}$ & $4(4 \%)$ \\
\hline Unknown & $2(2 \%)$ \\
\hline \multicolumn{2}{|l|}{ Histology } \\
\hline Infiltrating ductal carcinoma & $88(82 \%)$ \\
\hline Infiltrating lobular carcinoma & $9(8 \%)$ \\
\hline Tubular carcinoma & $3(3 \%)$ \\
\hline Medullar carcinoma & $3(3 \%)$ \\
\hline Tubulolobular carcinoma & $2(2 \%)$ \\
\hline Micro-invasive & $1(1 \%)$ \\
\hline Apocrine carcinoma & $1(1 \%)$ \\
\hline \multicolumn{2}{|l|}{ ER } \\
\hline Positive & $77(72 \%)$ \\
\hline Negative & $28(26 \%)$ \\
\hline Unknown & $2(2 \%)$ \\
\hline \multicolumn{2}{|l|}{ PR } \\
\hline Positive & $65(61 \%)$ \\
\hline Negative & $40(37 \%)$ \\
\hline Unknown & $2(2 \%)$ \\
\hline Mitose activity index & $20(1-88)$ \\
\hline
\end{tabular}

acute complication rate as $9.6 \%$.

Late complications were diffuse fibrosis $(\mathrm{n}=6$; grade $1)$, circumscript fibrosis $(n=13$; grade 1$)$, pain $(n=6$; grade 1$)$, atrophy $(\mathrm{n}=1$; grade 1$)$, asymptomatic lymphedema $(n=2$, grade 1$)$, symptomatic lymphedema $(n=$ 2 , grade 2 ), erysipelas $(n=1$; grade 1$)$, deformated breast without fibrosis ( $\mathrm{n}=1$; grade 1$)$, circumscript teleangiectasia $(\mathrm{n}=6$; grade 1$)$, chronic breast abscess $(\mathrm{n}=1$, grade 3). Excluding the lymphedema cases, which all occurred after axillary lymph node dissection, overall late complication rate was $35 \%$. Toxicity is summarized in Table 2.

\subsection{Follow-Up and Survival}

Excluding those patients that underwent ablative surgery because of tumor-positive margins left 100 patients eligible for survival analysis. Median follow-up of the patients still alive was 6.8 years (range 2.0 - 14.8). During follow-up three patients $(2.9 \%)$ developed a second primary contralateral breast cancer, which, in one patient was again treated according to the same treatment protocol. Three patients $(2.8 \%)$ developed a local recurrence in the breast. Disease-, and treatment-related characteristics of these patients are detailed in Table 3 . All patients with local recurrences were treated with salvage mastectomy. There were no axillary or supraclavicular recur-

Table 2. Treatment related toxicity of WLE + whole breast radiation + brachytherapy boost of 107 breasts in 105 patients, graded by the Common Terminology Criteria for Adverse Events (CTCAE), third edition. Because nine patients had more than one complication, the total number of complications is higher than the total number of patients with treatment related complications.

\begin{tabular}{lllll}
\hline \multicolumn{1}{c}{ CTCAE Grade } & 1 & 2 & 3 & 4 \\
\hline Acute complication & & & \\
Infection of lumpectomy site & 4 & & \\
Hematoma & 3 & & \\
Axillary wound infection & & 6 & \\
Pain & 1 & & \\
Sterile mastitis & 2 & & \\
Venous bleeding & & & 1 \\
Late complication & & & \\
Fibrosis & 19 & & \\
Chronic breast abscess & & \\
Pain & 6 & \\
Atrophy & 1 & \\
Asymptomatic lymphedema & 1 & \\
Symptomatic lymphedema & 1 & \\
Breast deformation & 1 & \\
Circumscript teleangiectasia & 5 & \\
\hline
\end{tabular}


Table 3. Characteristics of patients with true in field recurrences. IDC = invasive ductal carcinoma. DCIS = ductal carcinoma in situ. $\mathrm{ER}=$ estrogen receptor. $\mathrm{PR}=$ progesterone receptor. $\mathrm{TTR}=$ time to recurrence. $\mathrm{CMF}=$ cyclophosphamide, methotrexate and fluorouracil 5-FU. TAC = taxotere, adriamycin and cyclophosphamide. Primary mastectomy was performed after irradical wide local excision. Secundary mastectomy was performed after histologically proven recurrence.

\begin{tabular}{ccccccccccccccccc}
\hline Age $(\mathrm{y})$ & Size $(\mathrm{mm})$ & Tumor Type & Grade & DCIS & ER/PR & Resection & TTR (y) & T-stage & N-stage & $\begin{array}{c}\text { Systemic } \\
\text { therapy }\end{array}$ \\
\hline 54 & 18 & IDC & Poorly & Yes & $-/-$ & Radical & 9.3 & 2 & CMF \\
66 & 6 & IDC & Moderately & No & $+/+$ & Radical & 9.4 & 1 & 0 & None & Radical & 3.3 & 2 & 0 \\
40 & 27 & Lobular & Unknown & No & $+/+$ & None \\
\hline
\end{tabular}

rences. Eighteen patients $(17 \%)$ developed distant metastases and eleven succumbed to their disease. The projected five- and ten-year local recurrence free survival rate was $99 \%$ and $91 \%$, respectively (Figure 1). Fiveand ten-year disease-specific survival was $95 \%$ and $84 \%$, respectively (Figure 2). Finally, five- and ten-year overall survival was $92 \%$ and $78 \%$, respectively (Figure 3).

\section{Discussion}

The primary aim of the present study was to evaluate the outcome of boost given by mean of brachytherapy as an adjuvant to whole breast irradiation for the treatment of early stage breast cancer. The projected local recurrence free survival proved to be $99 \%$ and $91 \%$ after 5 and 10 years, respectively.

The EORTC prospective randomised multi-centre trial 22881/10882 investigating the role of a boost dose in breast conserving therapy demonstrated a significantly better local control rate with the higher radiotherapy dose. Patients were randomised to receive a boost dose of 16 Gy to the primary tumour bed after microscopically complete tumorectomy and 50 Gy whole breast irradiation. Of the 2661 patients, 9\% received the boost with interstitial brachytherapy. At 5 years, local recurrences were seen in 6 of the 225 patients after an interstitial boost (2.5\%). [13] Therefore, our results our comparable to that reported in literature, indicating that interstitial brachytherapy is a good alternative to the conventional radiation schemes. To our knowledge, no randomized trials have been conducted comparing whole breast radiation plus external beam boost to whole breast radiation plus brachytherapy boost. Nevertheless, among the patients receiving interstitial boost brachytherapy in the present study, only three true local recurrences occurred (2.8\%), thereby achieving excellent local control with intraoperatively placed needles for interstitial brachytherapy. This is supported by the prolonged period of follow up in our study.

Higher irradiation doses increases the risk of fibrosis and worsen cosmetic outcome [14]. In the present study, treatment-related toxicity was mild, since only one patient had grade 3 toxicity (hemorrhage after removal of the catheters requiring surgical exploration in theatre. However, 22 cases of fibrosis were encountered. At fol-

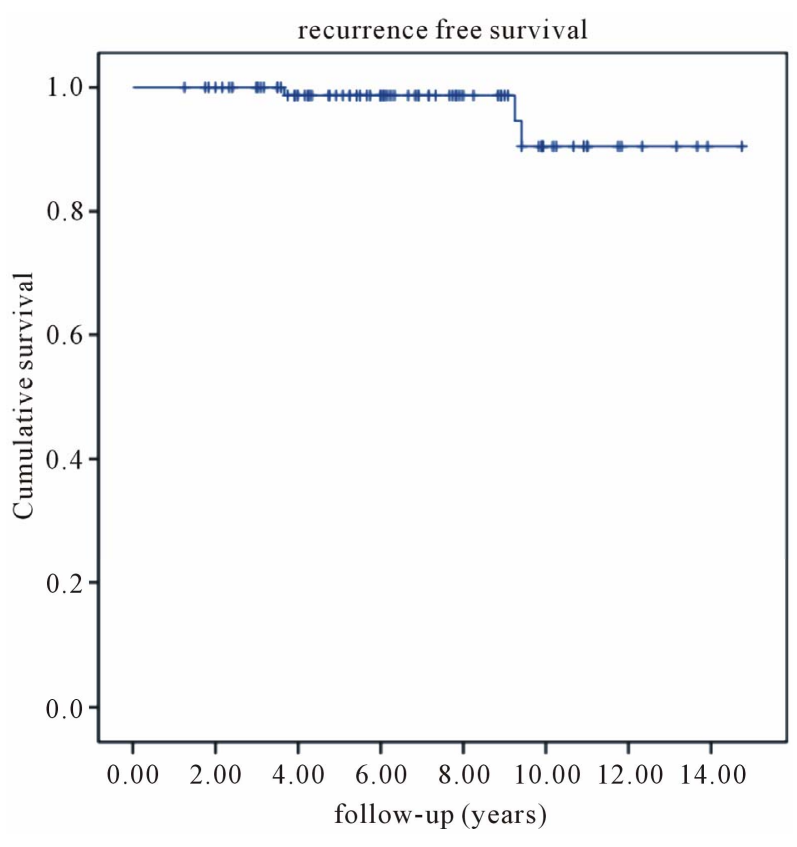

Figure 1. Kaplan-Meier estimates of recurrence free survival among patients with primary resectable breast cancer treated with wide local excision follow by iridium-based brachytherapy.

low up of the afore mentioned EORTC trial, technique of boost delivery (a.o. interstitial boost) was not significantly associated with an increased risk of fibrosis. The risk of fibrosis significantly increased with increasing maximum whole breast irradiation dose and with concomitant chemotherapy, but was independent of age. In the boost arm, the risk further increased if patients had post-operative breast edema or hematoma, but it decreased if WBI was given with more than $6 \mathrm{MV}$ photons [15]. We have no specific explanation for the rate of fibrosis in our study.

The main advantage of interstitial brachytherapy is that is allows precise deposition of radiation energy due to visualization of the excision site at the time of intra-operative implantation. Another advantage of interstitial brachytherapy given as a boost in BCT, is that total time of boost application is reduced in comparison to external beam technique.

The boost radiation dose can be applied either with 


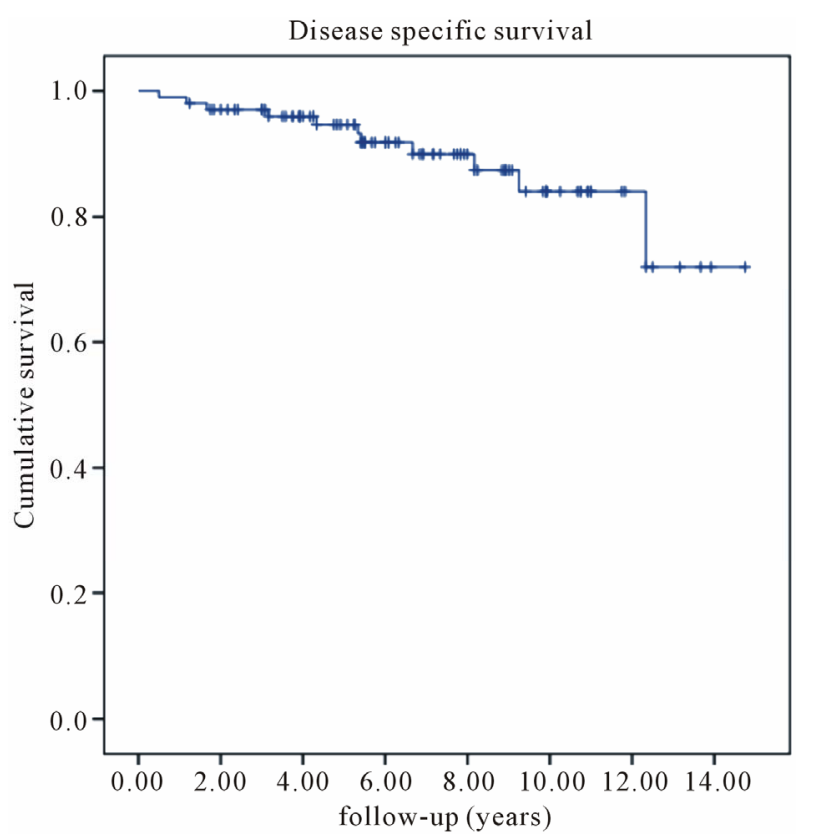

Figure 2. Kaplan-Meier estimates of disease specific survival among patients with primary resectable breast cancer treated with wide local excision follow by iridium-based brachytherapy.

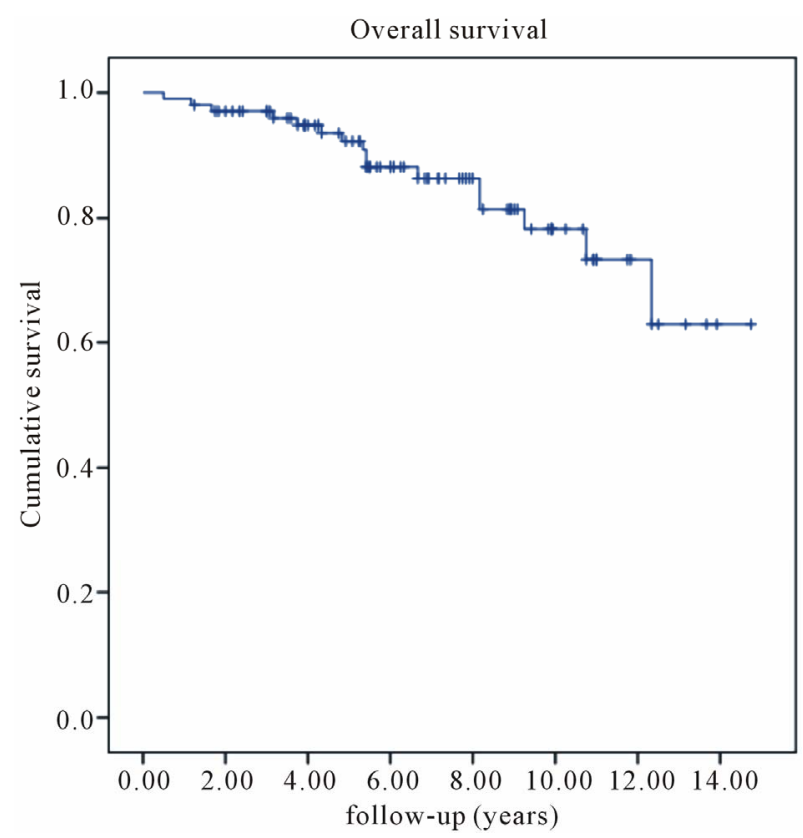

Figure 3. Kaplan-Meier estimates of overall survival among all patients with primary resectable breast cancer treated with wide local excision follow by iridium-based brachytherapy.

external beam, fractionated or with brachytherapy.

The main disadvantage of this procedure is the fact that the final histological reports concerning completeness of resection are not available during the boost treatment. In the present series, five out of 65 patients
(7.7\%) had tumor-positive resection margins, necessitateing secondary surgery.

In conclusion, a very close collaboration between a surgeon and radiation oncologist is necessary. We managed to introduce this procedure in one of our hospitals and decided to report on our results. We observed that interstitial brachytherapy has low local recurrence rate with mild treatment related toxicity. Supported by the long follow-up, the present study confirms that brachytherapy is a good alternative to conventional radiation boost as a part of breast irradiation in breast conserving treatment in early stage breast cancer.

\section{REFERENCES}

[1] J. Borger, H. Kemperman, A. Hart, H. Peterse, D. J. van and H. Bartelink, "Risk Factors in Breast-Conservation Therapy," Journal of Clinical Oncology, Vol. 12, No. 4, 1994, pp. 653-660.

[2] A. de la Rochefordiere, B. Asselain, F. Campana, S. M. Scholl, J. Fenton, J. R. Vilcoq, J. C. Durand, P. Pouillart, H. Magdelenat and A. Fourquet, "Age as Prognostic Factor in Premenopausal Breast Carcinoma," Lancet, Vol. 341, No. 8852, 1993, pp. 1039-1043. doi:10.1016/0140-6736(93)92407-K

[3] P. H. Elkhuizen, A. C. Voogd, L. C. van den Broek, I. T. Tan, H. C. van Houwelingen, J. W. Leer and M. J. van de Vijver, "Risk Factors for Local Recurrence after BreastConserving Therapy for Invasive Carcinomas: A CaseControl Study of Histological Factors and Alterations in Oncogene Expression," International Journal of Radiation Oncology*Biology*Physics, Vol. 45, No. 1, 1999, pp. 73-83.

[4] J. M. Kurtz, J. Jacquemier, R. Amalric, H. Brandone, Y. Ayme, D. Hans, C. Bressac, J. Roth and J. M. Spitalier, "Risk Factors for Breast Recurrence in Premenopausal and Postmenopausal Patients with Ductal Cancers Treated by Conservation Therapy," Cancer, Vol. 65, No. 8, 1990, pp. $1867-1878$. doi:10.1002/1097-0142(19900415)65:8<1867::AID-CNC R2820650833>3.0.CO;2-I

[5] A. Stotter, E. N. Atkinson, B. A. Fairston, M. McNeese, M. J. Oswald and C. M. Balch, "Survival Following Locoregional Recurrence after Breast Conservation Therapy for Cancer," Annals of Surgery, Vol. 212, No. 2, 1990, pp. 166-172. doi:10.1097/00000658-199008000-00009

[6] U. Veronesi, E. Marubini, L. Mariani, V. Galimberti, A. Luini, P. Veronesi, B. Salvadori and R. Zucali, "Radiotherapy after Breast-Conserving Surgery in Small Breast Carcinoma: Long-Term Results of a Randomized Trial," Annals of Oncology, Vol. 12, No. 7, 2001, pp. 997-1003. doi:10.1023/A:1011136326943

[7] J. M. Kurtz, R. Amalric, H. Brandone, Y. Ayme, J. Jacquemier, J. C. Pietra, D. Hans, J. F. Pollet, C. Bressac, J. M. Spitalier, "Local Recurrence after Breast-Conserving Surgery and Radiotherapy. Frequency, Time Course, and Prognosis," Cancer, Vol. 63, No. 10, 1989, pp. 19121917. 
doi:10.1002/1097-0142(19890515)63:10<1912::AID-CN CR2820631007>3.0.CO;2-Y

[8] S. Bensaleh, E. Bezak and M. Borg, "Review of MammoSite Brachytherapy: Advantages, Disadvantages and Clinical Outcomes," Acta Oncologica, Vol. 48, No. 4, 2009, pp. 487-494. doi:10.1080/02841860802537916

[9] R. Garza, K. Albuquerque and A. Sethi, "Lung and Cardiac Tissue Doses in Left Breast Cancer Patients Treated with Single-Source Breast Brachytherapy Compared to External Beam Tangent Fields," Brachytherapy, Vol. 5, No. 4, 2006, pp. 235-238. doi:10.1016/j.brachy.2006.08.001

[10] S. B. Edge and C. C. Compton, "The American Joint Committee on Cancer: The 7th Edition of the AJCC Cancer Staging Manual and the Future of TNM," Annals of Surgical Oncology, Vol. 17, No. 6, 2010, pp. 14711474.

[11] A. Trotti, A. D. Colevas, A. Setser, V. Rusch, D. Jaques, V. Budach, C. Langer, B. Murphy, R. Cumberlin, C. N. Coleman and P. Rubin, "CTCAE v3.0: Development of a Comprehensive Grading System for the Adverse Effects of Cancer Treatment," Seminars in Radiation Oncology, Vol. 13, No. 3, 2003, pp. 176-181. doi:10.1016/S1053-4296(03)00031-6

[12] B. Pierquin, A. Dutreix, C. H. Paine, D. Chassagne, G. Marinello and D. Ash, "The Paris System in Interstitial Radiation Therapy," Acta Radiologica: Oncology, Radiation, Physics, Biology, Vol. 17, No. 1, 1978, pp. 33-48.
[13] P. Poortmans, H. Bartelink, J. C. Horiot, H. Struikmans, W. Van Den Bogaert, A. Fourquet, J. Jager, W. Hoogenraad, P. Rodrigus, C. Warlam-Rodenhuis, L. Collette and M. Pierart, "The Influence of the Boost Technique on Local Control in Breast Conserving Treatment in the EORTC 'Boost versus No Boost' Randomised Trial,' Radiotherapy \& Oncology, Vol. 72, No. 1, 2004, pp. 25-33. doi:10.1016/j.radonc.2004.03.007

[14] D. Curran, J. P. van Dongen, N. K. Aaronson, G. Kiebert, I. S. Fentiman, F. Mignolet and H. Bartelink, "Quality of Life of Early-Stage Breast Cancer Patients Treated with Radical Mastectomy or Breast-Conserving Procedures: Results of EORTC Trial 10801. The European Organization for Research and Treatment of Cancer (EORTC), Breast Cancer Co-Operative Group (BCCG)," European Journal of Cancer, Vol. 34, No. 3, 1998, pp. 307-314. doi:10.1016/S0959-8049(97)00312-2

[15] S. Collette, L. Collette, T. Budiharto, J. C. Horiot, P. M. Poortmans, H. Struikmans, W. Van Den Bogaert, A. Fourquet, J. J. Jager, W. Hoogenraad, R. P. Mueller, J. Kurtz, D. A. Morgan, J. B. Dubois, E. Salamon, R. Mirimanoff, M. Bolla, M. Van der Hulst, C. C. Warlam-Rodenhuis and H. Bartelink, "Predictors of the Risk of Fibrosis at 10 Years after Breast Conserving Therapy for Early Breast Cancer: A Study Based on the EORTC Trial 22881-10882 'Boost versus No Boost'," European Journal of Cancer, Vol. 44, No. 17, 2008, pp. 2587-2599. doi:10.1016/j.ejca.2008.07.032 